\title{
Lateral Phase Separation in Grafted Diblock Copolymer Films
}

\author{
Benjamin M. D. O’Driscoll,,${ }^{\dagger}{ }^{\dagger}$ Guy H. Griffiths, ${ }^{\ddagger}$ Mark W. Matsen, ${ }^{\ddagger}$ Sébastien Perrier, ${ }^{\S}$ \\ Vincent Ladmiral, ${ }^{\S}$ and Ian W. Hamley ${ }^{\dagger}$ \\ ${ }^{\dagger}$ Department of Chemistry, University of Reading, Whiteknights, Reading, RG6 6AD, U.K., ${ }^{\star}$ Department of \\ Mathematics, University of Reading, Whiteknights, Reading, RG6 6AX, U.K., and ${ }^{\S}$ Key Centre for \\ Polymers and Colloids, School of Chemistry, University of Sydney, NSW, 2006, Australia
}

Received May 17, 2010; Revised Manuscript Received August 3, 2010

\begin{abstract}
Tethered films of polystyrene-block-poly(methyl methacrylate) copolymers of varying composition and molecular weight were investigated using atomic force microscopy and the observed structures compared with theoretical predictions. Although the experimental results were in qualitative agreement with the theory, there was significant quantitative variation. This was attributed to the presence of solvent in the films prior to and during annealing, a hypothesis supported by new preliminary calculations reported here. Solvent exchange experiments (where a good solvent for both polymer blocks was gradually replaced by a selective solvent), were also performed on the films. This procedure generated textured films in which the structure was defined by miscibility of the polymer blocks with the second solvent.
\end{abstract}

\section{Introduction}

In both experimental and theoretical studies, it has been shown that diblock copolymers have rich phase behavior in the bulk, ${ }^{1-3}$ with the phase that is formed being determined by the molecular weight of the polymer $\left(M_{\mathrm{n}}\right)$ (degree of polymerization, $\left.N\right)$, the composition of the polymer $(f)$ and the Flory-Huggins interaction parameter $(\chi)$. Moreover, when cast as thin films these polymers may present a surface with defined chemical patterning. ${ }^{1}$ Such films have been used to selectively bind proteins to the surface and many other applications, including nanolithographic patterning and membrane filtration, have been proposed. ${ }^{4-7}$

Films of block copolymers grafted to the surface have received relatively less attention. ${ }^{8,9}$ Grafting of the polymer chain offers several advantages over untethered films, including greater resistance to abrasion and prevention of dewetting. However, in reducing the degrees of freedom available to the polymer chains the grafting process also has a significant impact on the phase behavior of the polymer.

In a recent publication by Matsen and Griffiths self-consistent field theory (SCFT) calculations were used to determine the equilibrium phase behavior of tethered copolymer films. ${ }^{10}$ In total only four phases are predicted to form: uniform, hexagonal, stripe and inverse hexagonal (Figure 1).

As with bulk systems the phase behavior is dependent on $\chi, N$, and $f$; however for the tethered systems, it is also necessary to consider the grafting density of the polymer $(\sigma)$ and the difference in the surface affinity of two blocks $(\Lambda)^{10}$

$$
\Lambda \equiv \frac{\left(\gamma_{B}-\gamma_{A}\right) v}{k_{\mathrm{B}} T R_{0}}
$$

where $\gamma_{A}$ and $\gamma_{B}$ are the surface tensions of the upper and lower block respectively, $k_{B}$ is Boltzmann's constant, $T$ is the temperature, $v$ is the monomer volume, and $R_{0}$ is the unperturbed mean free end-to-end length of the polymer.

The presence of these additional parameters increases the complexity of the phase space, complicating the determination

\footnotetext{
*Corresponding author. E-mail: b.odriscoll@reading.ac.uk.
}

of a generic phase diagram for grafted diblock films. Figure 2 shows the phase diagram for one specific system. In line with convention the combined (molecular weight independent) parameters $\chi N, \Lambda N$ and $L / a N^{1 / 2}$ are used, where $L$ is the film thickness, which is proportional to $\sigma$, and $a$ is the statistical segment length of the polymer (where $a N^{1 / 2}$ is equal to $R_{0}$ ).

As was noted by Matsen and Griffiths, ${ }^{10}$ the phase diagrams of tethered films are qualitatively similar to those of the bulk system. $^{2}$ The most prominent differences are the shift of the nonuniform phases to low untethered block compositions (generally $f<0.5$ ), an increase in the $\chi N$ value of the critical point, and a pronounced dependence on both $L / a N^{1 / 2}$ and $\Lambda N{ }^{10}$ As the grafting density increases, the area occupied by nonuniform phases in the phase diagram decreases and the phase boundaries shift. Having an upper block with a higher surface tension than the lower grafted block (yielding a negative $\Lambda N$ ) expands the area occupied by the nonuniform phases.

The phase diagrams reported by Matsen and Griffiths represent systems with moderate grafting densities and, as such, are the most appropriate for the grafting-to method used to prepare the polymer films in this study.

Importantly these phase diagrams are the first published for tethered diblock copolymer films. They are built on the model that has been used to generate the well-known phase diagram for bulk copolymer systems produced by Matsen and Bates, ${ }^{2}$ and as such represent the thermodynamic equilibrium phase behavior for these films. In performing this study we have aimed to verify and inform this theoretical work with experimental data. This was done by examining the phase behavior of a number of polystyrene-block-poly(methyl methacrylate) (PS- $b$-PMMA) films tethered to surfaces. In the first instance hydroxyl-terminated polymers were attached to bare silicon wafers, while in the second thiol-terminated polymers were attached to gold-coated silicon wafers. The phase behavior was examined with atomic force microscopy (AFM) and we have compared the observed results with the model systems. Where differences are observed we attribute these to the presence of solvent in the film, which new preliminary calculations show has a significant impact on the phase behavior.

Published on Web 09/13/2010

pubs.acs.org/Macromolecules 


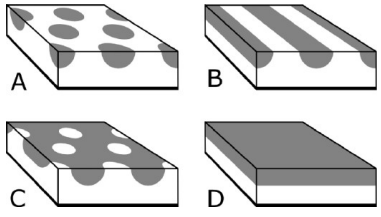

Figure 1. The accessible phases of tethered block copolymer films (a) hexagonal, (b) stripe, (c) inverse hexagonal, and (d) uniform. Here the grafted polymer block is white and the untethered block is gray.

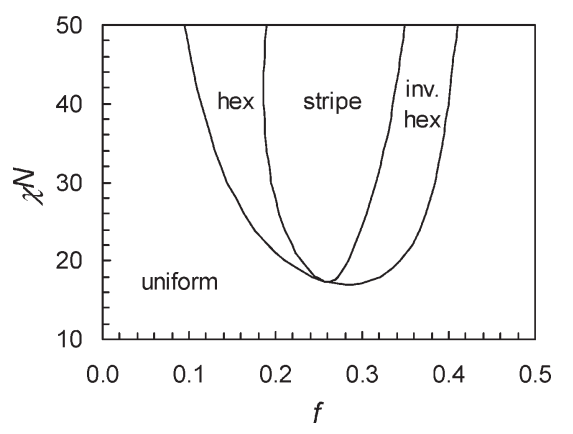

Figure 2. Equilibrium phase diagram of a grafted diblock copolymer film calculated for $L / a N^{1 / 2}=0.5$ and $\Lambda N=0$.

In a second series of experiments, we examine the structures formed when the environment around the films is gradually changed from one where the solvent is a good solvent for both blocks to one where it is selective for one block, (referred to here as solvent exchange), in the vein of the work by Zhao, Brittain, and co-workers. ${ }^{11,12}$

\section{Experimental Section}

The thiol-terminated PMMA- $b$-PS-SH polymers were synthesized by RAFT polymerization as previously described, ${ }^{13-16}$ while the hydroxyl-terminated, polystyrene-deuterated polymers, $d$ PS- $b$-PMMA-OH were purchased from Polymer Standards Service (Mainz, Germany). All remaining chemicals were purchased from Aldrich or Fisher, were of AR grade or better, and were used without further purification.

Polished silicon wafers were obtained from Prolog Semicor Ltd. (Ukraine). These were cleaned with a Piranha solution $(70 \%$ $\mathrm{H}_{2} \mathrm{SO}_{4}, 30 \% \mathrm{H}_{2} \mathrm{O}_{2}$ ) at $100{ }^{\circ} \mathrm{C}$ for $1 \mathrm{~h}$, washed with water and ethanol then dried under vacuum at $70{ }^{\circ} \mathrm{C}$. (Caution! Piranha solution can react violently with organic compounds.) Some wafers were sputter-coated with a $\sim 30 \mathrm{~nm}$ thick layer of gold.

$d$ PS- $b$-PMMA-OH films were prepared by spin-coating $2 \mathrm{wt} \%$ solutions in toluene at $2000 \mathrm{rpm}$ onto cleaned silicon wafers. Similarly PMMA- $b$-PS-SH films were prepared using 5 wt $\%$ solutions at $1000 \mathrm{rpm}$ on gold-coated wafers.

The $d$ PS- $b$-PMMA-OH films were annealed at $180{ }^{\circ} \mathrm{C}$ for 25 or $161 \mathrm{~h}$ under vacuum then the excess polymer was removed by sonication in neat toluene $(2 \times 15 \mathrm{~min})$ and the wafers dried under a stream of $\mathrm{N}_{2}$. The PMMA- $b$-PS-SH films were exposed to a saturated vapor of THF for $256 \mathrm{~h}$ (so-called solvent annealing) then soaked (with occasional gentle agitation) in neat toluene overnight, rinsed to remove the excess polymer and dried under a stream of $\mathrm{N}_{2}$.

Following initial annealing and washing the samples were treated in one of three ways:

Thermal Annealing. Some of the $d \mathrm{PS}-b-\mathrm{PMMA}-\mathrm{OH}$ films were annealed at $180{ }^{\circ} \mathrm{C}$ under vacuum for a further $6 \mathrm{~h}$.

Solvent Annealing. Likewise a number of PMMA- $b$-PS-SH films were exposed to toluene vapor for $48 \mathrm{~h}$.

Solvent Exchange. The remaining films were immersed in THF ( $d$ PS- $b$-PMMA-OH) or toluene (PMMA- $b$-PS-SH), both good solvents for the two polymers, then cyclohexane or acetone was added dropwise until the added solvent constituted $75 \%$ of
Table 1. Physical Parameters of the Polymers Examined in This Study

\begin{tabular}{lcccccc}
\hline \multicolumn{1}{c}{ polymer } & $M_{\mathrm{n}}(\mathrm{kg} / \mathrm{mol})$ & $\mathrm{PDI}^{a}$ & $f$ & $\chi N$ & $\Lambda N$ & $R_{0}(\mathrm{~nm})$ \\
\hline$d \mathrm{PS}_{13} \mathrm{PMMA}_{100} \mathrm{OH}$ & 113.0 & 1.07 & 0.10 & 41.3 & 1.24 & 21.8 \\
$d \mathrm{PS}_{33} \mathrm{PMMA}_{122} \mathrm{OH}$ & 155.0 & 1.12 & 0.19 & 56.0 & 1.47 & 25.5 \\
$d \mathrm{PS}_{47} \mathrm{PMMA}_{135} \mathrm{OH}$ & 182.0 & 1.14 & 0.24 & 65.4 & 1.60 & 27.6 \\
$d \mathrm{PS}_{62} \mathrm{PMMA}_{69} \mathrm{OH}$ & 131.0 & 1.07 & 0.45 & 46.0 & 1.38 & 23.3 \\
$\mathrm{PMMA}_{28} \mathrm{PS}_{101} \mathrm{SH}$ & 128.3 & 1.40 & 0.22 & 53.7 & -1.46 & 23.5 \\
$\mathrm{PMMA}_{52} \mathrm{PS}_{108} \mathrm{SH}$ & 160.0 & 1.44 & 0.33 & 67.2 & -1.60 & 26.3 \\
$\mathrm{PMMA}_{42} \mathrm{PS}_{84} \mathrm{SH}$ & 125.3 & 1.40 & 0.34 & 52.7 & -1.42 & 23.3 \\
$\mathrm{PMMA}_{72} \mathrm{PS}_{62} \mathrm{SH}$ & 133.3 & 1.38 & 0.55 & 56.5 & -1.43 & 23.9 \\
$\mathrm{PMMA}_{72} \mathrm{PS}_{31} \mathrm{SH}$ & 102.5 & 1.34 & 0.71 & 43.8 & -1.22 & 21.0 \\
${ }^{a} \mathrm{PDI}_{\text {denotes the polydispersity index. }}$ & & & &
\end{tabular}

the volume. The wafers were removed and dried under a stream of $\mathrm{N}_{2}$. A number of the $d \mathrm{PS}-b$-PMMA-OH films treated this way were subsequently thermally annealed at $180^{\circ} \mathrm{C}$ under vacuum for $3 \mathrm{~h}$.

AFM images were collected with a Veeco Explorer with a $2 \mu \mathrm{m}$ scanner in noncontact mode. AFM tips (Veeco) with $k=$ $20-80 \mathrm{~N} / \mathrm{m}$ and $f_{0}=130-320 \mathrm{kHz}$ were used with a set-point ratio of $50-60 \%$.

SEM images were collected with a FEI Quanta FEG 600 Environmental SEM in high vacuum mode.

Ellipsometry measurements were performed at the University of Surrey, U.K., on a J.A Woollam ellipsometer. The thin films were modeled as a single layer, however due to their small thicknesses the values of the Cauchy coefficients were fixed at $A=1.52$ and $B=0.001$.

\section{Results and Discussion}

The primary means of accessing different phases in the phase diagram of a given block copolymer system is to vary the composition. For tethered block copolymers it has been shown theoretically that the nonuniform phases are skewed toward diblock compositions where the upper, untethered block is smaller than the tethered block, i.e., $f<0.5{ }^{10}$ In this study we have examined nine polymers, seven of which have $f<0.5$. The specific parameters of the polymers examined are given in Table 1; the subscripts in each polymer designation give the approximate molecular weight (in $\mathrm{kg} / \mathrm{mol}$ ) of each block.

A notable result in Table 1 is the magnitude of the polymeric $\Lambda N$ values relative to the value used in one of the models $(\Lambda N=$ $-0.15)$ calculated by Matsen and Griffiths. ${ }^{10}$ This model value is equivalent to a surface tension difference $(\Delta \gamma)$ between the two blocks of $\sim 0.12 \mathrm{mN} / \mathrm{m}$ (calculation shown in Supporting Information), as opposed to $\Delta \gamma=1.0$ for the PS-PMMA system $\left(\gamma_{\mathrm{PS}}=40.2 \mathrm{mN} / \mathrm{m}\right.$ and $\left.\gamma_{\text {PMMA }}=41.2 \mathrm{mN} / \mathrm{m}\right) .{ }^{17}$ It is therefore apparent that this parameter, and hence the calculated phase diagram, is highly sensitive to the surface tensions of the two polymers blocks.

Regarding the other values in Table 1 , it should be noted that the polydispersity of block B makes a greater contribution to the total polydispersity due to the method of manufacture of both types of polymer. The values of $\chi,{ }^{18,19}$ and $R_{0},{ }^{20}$ were calculated from literature values.

Spin-coating was used to deposit the polymers onto the substrate surfaces and this generated films $\sim 70 \mathrm{~nm}$ thick for the $d$ PS- $b$-PMMA-OH samples, and $\sim 400 \mathrm{~nm}$ thick for the PMMA- $b$-PS-SH samples. These films were either thermally or solvent annealed to produce the tethered polymer films, with the untethered polymer being washed off following this first annealing process. To induce phase separation the tethered films were annealed a second time. For the $d \mathrm{PS}-\mathrm{PMMA}-\mathrm{OH}$ films this second thermal annealing step is expected to drive off any residual solvent during the annealing process, while for the PMMAPS-SH films the solvent was left to evaporate away under ambient conditions at the end of the second solvent annealing step. 
Table 2. Parameters Relating to the Film Thickness of the Tethered Polymer Films

\begin{tabular}{lrcc}
\hline \multicolumn{1}{c}{ polymer } & \multicolumn{1}{c}{$L(\mathrm{~nm})$} & $L / a N^{1 / 2}$ & $\sigma\left(\mathrm{nm}^{-2}\right)$ \\
\hline$d \mathrm{PS}_{13} \mathrm{PMMA}_{100} \mathrm{OH}$ & $7.32 \pm 0.02$ & 0.34 & 0.045 \\
$d \mathrm{PS}_{33} \mathrm{PMMA}_{122} \mathrm{OH}$ & $9.42 \pm 0.02$ & 0.37 & 0.042 \\
$d \mathrm{PS}_{47} \mathrm{PMMA}_{135} \mathrm{OH}$ & $11.20 \pm 0.01$ & 0.41 & 0.042 \\
$d \mathrm{PS}_{62} \mathrm{PMMA}_{69} \mathrm{OH}$ & $14.48 \pm 0.04$ & 0.62 & 0.075 \\
$\mathrm{PMMA}_{2} \mathrm{PS}_{101} \mathrm{SH}$ & $8.7 \pm 1.2$ & 0.37 & 0.042 \\
$\mathrm{PMMA}_{52} \mathrm{PS}_{108} \mathrm{SH}$ & $13.3 \pm 1.2$ & 0.51 & 0.053 \\
$\mathrm{PMMA}_{42} \mathrm{PS}_{84} \mathrm{SH}$ & $8.0 \pm 1.2$ & 0.34 & 0.041 \\
$\mathrm{PMMA}_{72} \mathrm{PS}_{62} \mathrm{SH}$ & $7.7 \pm 1.2$ & 0.32 & 0.038 \\
PMMA $_{72} \mathrm{PS}_{31} \mathrm{SH}$ & $10.4 \pm 1.2$ & 0.50 & 0.068
\end{tabular}
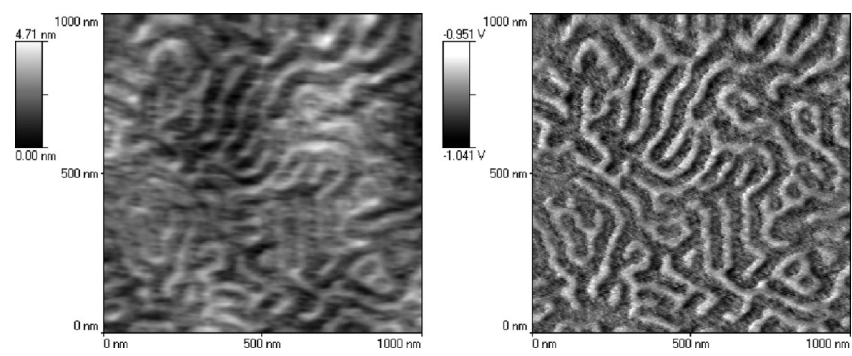

Figure 3. AFM topographic (left) and phase (right) images of a $\sim 70 \mathrm{~nm}$ thick $d \mathrm{PS}_{62} \mathrm{PMMA}_{69} \mathrm{OH}$ film after annealing for $25 \mathrm{~h}$.

The film thicknesses of the washed $d \mathrm{PS}-b$-PMMA-OH and PMMA- $b$-PS-SH samples, as measured by ellipsometry or AFM line scans (Supporting Information), are presented in Table 2.

Also listed in Table 2 are the calculated values of $L / a N^{1 / 2}$ and $\sigma$, which were determined using known values for the monomer volumes of PS and PMMA. ${ }^{21}$

A notable result evident in Table 2 is the different grafting densities of the films. This can be explained by the preference of the silicon oxide surface to be wetted by PMMA rather than $d \mathrm{PS}$; if only the PMMA segments are in contact with the surface then the probability of the end-group being in contact (and reacting) with the surface will be inversely proportional to the size of the PMMA block and independent of $M_{\mathrm{n}}$. The grafting densities should thus increase in the order $d \mathrm{PS}_{62} \mathrm{PMMA}_{69} \mathrm{OH}>$ $d \mathrm{PS}_{13} \mathrm{PMMA}_{100} \mathrm{OH}>d \mathrm{PS}_{33} \mathrm{PMMA}_{122} \mathrm{OH}>d \mathrm{PS}_{47} \mathrm{PMMA}_{135} \mathrm{OH}$, a trend that was generally observed here.

Annealing time did not appear to have a strong influence on the grafting density; the $d \mathrm{PS}-b$-PMMA-OH films reported in this paper were annealed for either 25 or $161 \mathrm{~h}$ with no apparent trend in the grafting densities.

Samples Following the First Annealing Step. AFM images of the annealed films were taken prior to removal of the excess, untethered polymer, with Figure 3 showing the results for the $d \mathrm{PS}_{62} \mathrm{PMMA}_{69} \mathrm{OH}$ film (the other results are given in Figure 2 of the Supporting Information). These images showed a transition from a disordered phase $\left(d \mathrm{PS}_{13} \mathrm{PMMA}_{100} \mathrm{OH}\right)$, to increasingly long and thin stripes of PMMA in a matrix of PS as the fraction of PS increases. (Note for all the AFM phase images PMMA appears as the brighter phase. ${ }^{22-24}$ ) The phase diagram reported for diblock copolymers in the bulk predicts that $d \mathrm{PS}_{13} \mathrm{PMMA}_{100} \mathrm{OH}$ will be disordered, both $d \mathrm{PS}_{33} \mathrm{PMMA}_{122} \mathrm{OH}$ and $d \mathrm{PS}_{47} \mathrm{PMMA}_{135} \mathrm{OH}$ will form a cylindrical phase, and $d \mathrm{PS}_{62} \mathrm{PMMA}_{69} \mathrm{OH}$ will form a lamellar phase. $^{2}$ This is consistent with the AFM results reported here.

For the $d \mathrm{PS}_{33} \mathrm{PMMA}_{122} \mathrm{OH}, d \mathrm{PS}_{47} \mathrm{PMMA}_{135} \mathrm{OH}$ and $d \mathrm{PS}_{62} \mathrm{PMMA}_{69} \mathrm{OH}$ films it was possible to determine (by doing a fast Fourier transform (FFT) of the phase images), lateral domain spacings of $70 \mathrm{~nm}, 69$ and $58 \mathrm{~nm}$, respectively. These values are roughly twice the $R_{0}$ values given for these polymers in Table 1, indicating lateral stretching of the chains.

As has been noted previously, ${ }^{23,25,26}$ the topographic and phase images generated by AFM are not decoupled from
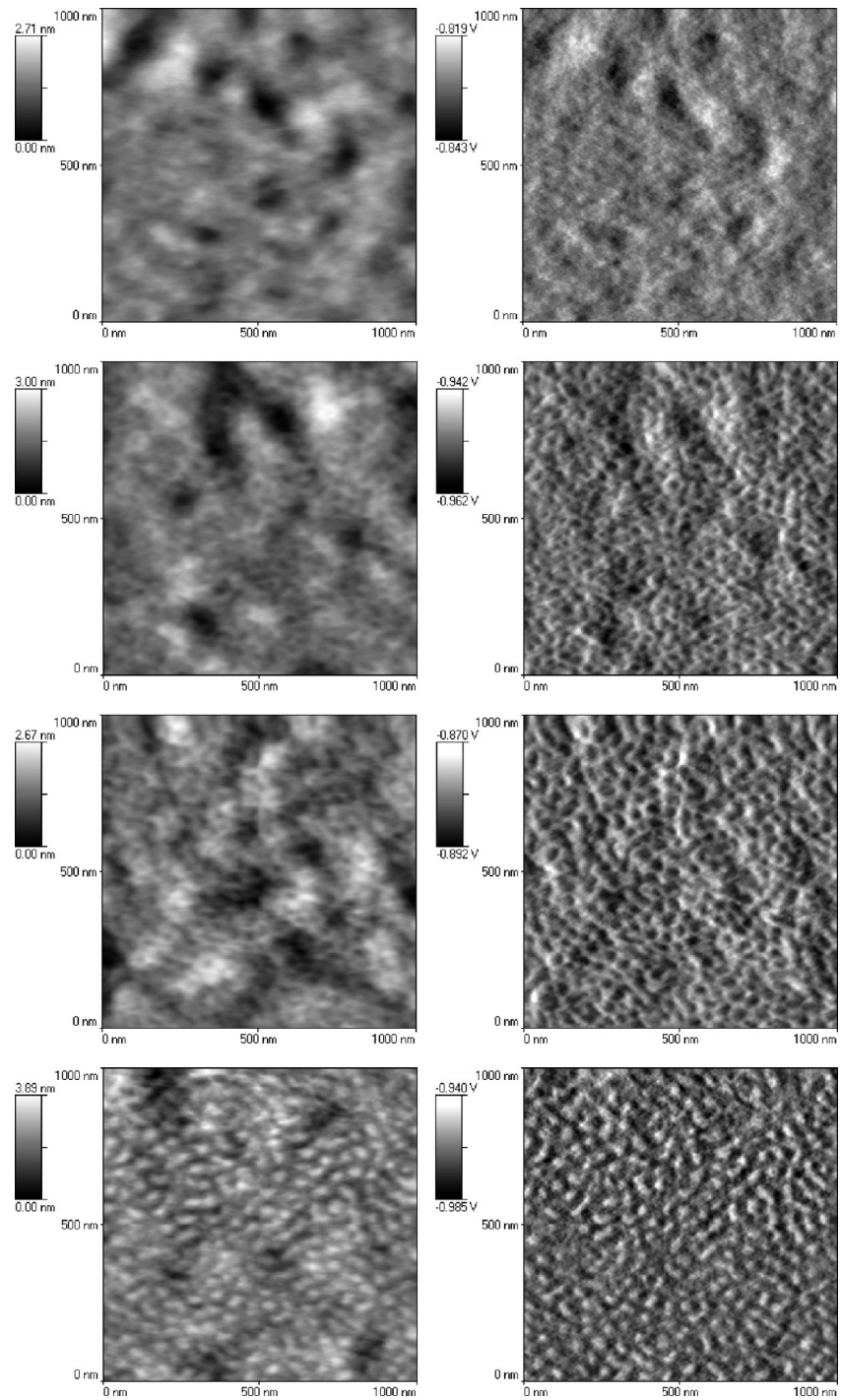

Figure 4. Topographic (left) and phase (right) AFM images of (from top) $d \mathrm{PS}_{13} \mathrm{PMMA}_{100} \mathrm{OH}, d \mathrm{PS}_{33} \mathrm{PMMA}_{122} \mathrm{OH}, d \mathrm{PS}_{47} \mathrm{PMMA}_{135} \mathrm{OH}$, and $d \mathrm{PS}_{62} \mathrm{PMMA}_{69} \mathrm{OH}$.

each other. It is therefore common to see artificial height variations in the topographic images that are due to changes in the mechanical properties of the underlying polymer (as measured by phase imaging). Equally, pronounced changes in the height may impact upon the phase image. In Figure 3 it is probable therefore that the low ridges $(\sim 2 \mathrm{~nm}$ high and $\sim 25 \mathrm{~nm}$ wide) observed in the topographic image that correspond to the stripes in the phase image represent artificial height changes, while the two broad ( $\sim 300 \mathrm{~nm}$ wide) stripes represent true height variations across the sample.

Samples Following Washing and the Second Annealing Step. Following the second thermal annealing step on the $d \mathrm{PS}-b$-PMMA-OH films there is a noticeable change in the nature of the phases present (Figure 4). Rather than the bulk phases observed in the thicker films these films present both the normal hexagonal phase $\left(d \mathrm{PS}_{33} \mathrm{PMMA}_{122} \mathrm{OH}\right.$ and $\left.d \mathrm{PS}_{47} \mathrm{PMMA}_{135} \mathrm{OH}\right)$, and the inverted hexagonal phase $\left(d \mathrm{PS}_{62} \mathrm{PMMA}_{69} \mathrm{OH}\right)$ of the tethered polymer system. The $d \mathrm{PS}_{13} \mathrm{PMMA}_{100} \mathrm{OH}$ film again presented a disordered/ uniform structure - in tethered films there is no distinction between the uniform and disordered phases.

The lack of long-range order in the phase-separated films is unsurprising as the nonequilibrium effects inherent to 


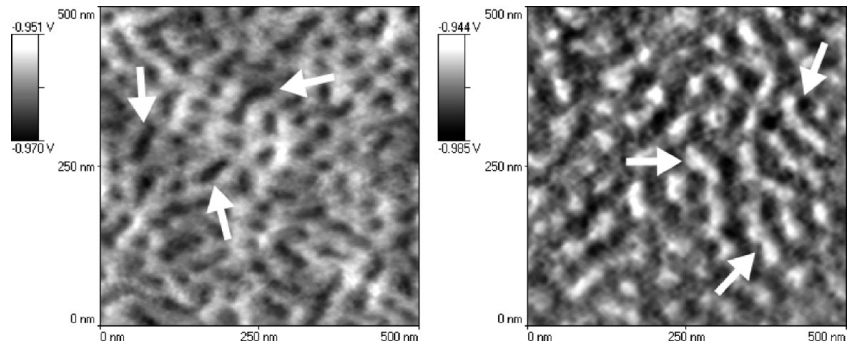

Figure 5. AFM phase images of a section of a $d \mathrm{PS}_{47} \mathrm{PMMA}_{135} \mathrm{OH}$ film (left) and a $d \mathrm{PS}_{62} \mathrm{PMMA}_{69} \mathrm{OH}$ film (right). The arrows indicate areas with elongated domains.

block copolymer systems are likely to be further exacerbated for tethered copolymers. ${ }^{10}$ This has been highlighted by single-chain in mean-field calculations of the related system of a tethered diblock copolymer swollen with solvent.

The presence of the disordered, hexagonal and inverse hexagonal morphologies is qualitatively consistent with the theoretical model, however a number of the films display a different phase to that expected. ${ }^{10}$ In particular, with reference to the phase diagram published by Matsen and Griffiths at $L / a N^{1 / 2}=$ 0.6 and $\Lambda N=0,{ }^{10}$ it was expected that the $d \mathrm{PS}_{62} \mathrm{PMMA}_{69} \mathrm{OH}$ film would present a uniform structure instead of the observed inverse hexagonal structure. This variance is magnified by the fact that the comparison does not take into account the very large $\Lambda N$ value for the $d \mathrm{PS}_{62} \mathrm{PMMA}_{69} \mathrm{OH}$ film (Table 1), which is expected to shrink the range over which nonuniform phases are observed. Indeed, though no specific calculations have been performed, it is possible that large positive $\Lambda N$ values will prevent the formation of any nonuniform phases as the relatively low surface tension of the upper block would be likely to dominate the free energy.

It is apparent therefore that there is a significant variable that needs to be included to reconcile the observed variation between the experimental and theoretical results. We believe that this is related to the presence of trapped solvent in the film, as is discussed later in the text.

FFT analysis performed on the AFM images of $d \mathrm{PS}_{33^{-}}$ $\mathrm{PMMA}_{122} \mathrm{OH}, d \mathrm{PS}_{47} \mathrm{PMMA}_{135} \mathrm{OH}$, and $d \mathrm{PS}_{62} \mathrm{PMMA}_{69} \mathrm{OH}$ shown in Figure 4, generated periodicity values of 40, 45, and $51 \mathrm{~nm}$, respectively. These periodicity values are smaller than those determined for the as-cast films (Figure 2), which is consistent with the asymmetric profile of the films normal to the substrate and subsequently provides indirect evidence for pseudo-2D microphase separation.

In the theoretical model it was also determined that increasing the grafting density will shift the onset of nonuniform phases to higher $\chi N$ (or molecular weight for a given diblock system), and alter the points at which the phase transitions occur. ${ }^{10}$ This effect was observed in a third section of the $d \mathrm{PS}_{47} \mathrm{PMMA}_{135} \mathrm{OH}$ film (Figure 5), which shows the coexistence of hexagonal-type and stripe-type features. Equally, the coexistence of inverted hexagonal-type and stripe-type features was observed in the $d \mathrm{PS}_{62} \mathrm{PMMA}_{69} \mathrm{OH}$ sample.

For high grafting densities it is predicted that the minimum molecular weight (or more specifically $\chi N$ ) at which nonuniform phases are allowed increases. ${ }^{10}$ Such a state was not observed in these films, however it was previously observed in PMMA- $b$-PS films prepared via a grafting-to method, which had a grafting density of $0.34 \mathrm{~nm}^{-2} .^{23}$

Samples Following Solvent Annealing. As is shown in Table 1, five PMMA- $b$-PS-SH polymers were tethered to gold-coated substrates via thiol-gold bonds and treated with a solvent annealing procedure. Because of the method of preparation the gold films were not entirely flat (Figure 6)

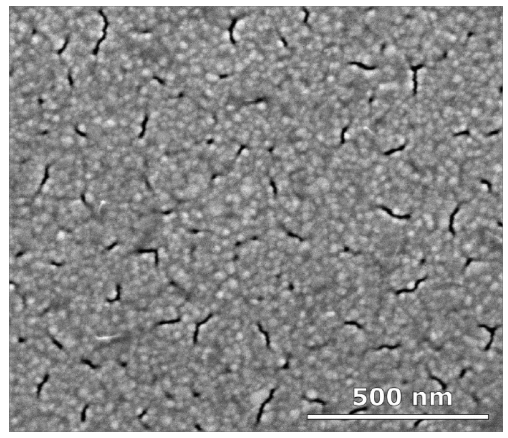

Figure 6. SEM image of a sputter-coated gold surface.

and showed height variations (of several nanometers) over small scales and isolated crevasses over larger scales.

This texturing is largely absent from the annealed polymer films (Figure 7), which is consistent with the polymer film minimizing the surface energy upon drying of the solvent. The ability of diblock copolymers to smooth out topographic defects of the substrate has previously been reported. ${ }^{27,28}$ Equally, Sivaniah et al. demonstrated that the phase-separated structures of free-standing block copolymer films can be affected by the roughness of the substrate and it is therefore likely that the substrate roughness will have an effect on the structure of the tethered films reported here. ${ }^{27}$

The $\mathrm{PMMA}_{28} \mathrm{PS}_{101} \mathrm{SH}, \mathrm{PMMA}_{52} \mathrm{PS}_{108} \mathrm{SH}$, and $\mathrm{PMMA}_{42-}$ $\mathrm{PS}_{84} \mathrm{SH}$ samples all display hexagonal ordering. (Note: PMMA rather than PS is the upper block in these films.) The $\mathrm{PMMA}_{72} \mathrm{PS}_{62} \mathrm{SH}$ sample presents a somewhat confused phase pattern that may be a poorly ordered stripe structure, while the $\mathrm{PMMA}_{72} \mathrm{PS}_{31} \mathrm{SH}$ film displays a uniform phase.

As with the $d$ PS- $b$-PMMA-OH samples some of the PMMA- $b$-PS-SH samples also display morphologies which differ from those expected (for $\Lambda N=0$ ). In particular, the $\mathrm{PMMA}_{52} \mathrm{PS}_{108} \mathrm{SH}$ sample, which has a $L / a N^{1 / 2}$ value of 0.51 , displays a hexagonal phase rather than stripe phase that would be expected from the $L / a N^{1 / 2}=0.5, \Lambda N=0$ phase diagram in Figure 2.

Relative to the $d \mathrm{PS}-b$-PMMA-OH samples at low composition the PMMA- $b$-PS-SH samples showed similar phase behavior; both the $\mathrm{PMMA}_{28} \mathrm{PS}_{101} \mathrm{SH}$ and $d \mathrm{PS}_{33^{-}}$ $\mathrm{PMMA}_{122} \mathrm{OH}$ films, where the polymers have very similar grafting densities, compositions and size, formed the hexagonal phase. However at higher compositions there was notable variance: the $d \mathrm{PS}_{47} \mathrm{PMMA}_{135} \mathrm{OH}$ film $(f=0.24)$ formed the inverse hexagonal phase, while the $\mathrm{PMMA}_{42} \mathrm{PS}_{84} \mathrm{SH}$ film $(f=0.34)$ formed the hexagonal phase.

As was stated above the substrate used for the PMMAPS-SH films showed some topographic variation over scales commensurate with the periodicity of the phase-separated structure, but this was not dramatically large and we considered it unlikely to be the sole cause of the variation between the PMMA-PS-SH and $d \mathrm{PS}-\mathrm{PMMA}-\mathrm{OH}$ films. Equally, although the large, negative values of $\Lambda N$ for the PMMA-PS-SH films were expected to expand the range over which the nonuniform phases were observed, the results for the $d \mathrm{PS}-\mathrm{PMMA}-\mathrm{OH}$ films showed that $\Lambda N$ could not account for the observed changes either.

The only significant difference between the two films was the annealing process; the PMMA-PS-SH films were annealed in a solvent atmosphere and so would have had substantial amounts of solvent incorporated into the films during annealing. The principle difficulty with attributing the observed changes to the presence of solvent was that the $d \mathrm{PS}-\mathrm{PMMA}-\mathrm{OH}$ films, which should have no solvent in the film post annealing, also 



Figure 7. AFM topographic (left) and phase (right) images of (topto-bottom): $\mathrm{PMMA}_{28} \mathrm{PS}_{101} \mathrm{SH}, \mathrm{PMMA}_{52} \mathrm{PS}_{108} \mathrm{SH}, \mathrm{PMMA}_{42} \mathrm{PS}_{84} \mathrm{SH}$, $\mathrm{PMMA}_{72} \mathrm{PS}_{62} \mathrm{SH}$, and $\mathrm{PMMA}_{72} \mathrm{PS}_{31} \mathrm{SH}$.

showed substantial divergence from the theoretical model. The resolution of this apparent paradox is discussed below.

The poorly defined ordering in the $\mathrm{PMMA}_{72} \mathrm{PS}_{62} \mathrm{SH}$ sample may be a sign of the very low grafting density of this film, the lowest of all the films examined. This could allow for interactions between the upper (PMMA) block and the substrate (an interaction not included in the theoretical model), which in turn could significantly disrupt the expected phase separation behavior.

Again there is a strong correlation between the domain spacing and the size of the upper block. FFT analysis of the AFM images yielded periodicity values of $34 \mathrm{~nm}$ for $\mathrm{PMMA}_{28} \mathrm{PS}_{101} \mathrm{SH} ; 58 \mathrm{~nm}$ for $\mathrm{PMMA}_{52} \mathrm{PS}_{108} \mathrm{SH} ; 42 \mathrm{~nm}$ for $\mathrm{PMMA}_{42} \mathrm{PS}_{84} \mathrm{SH}$, and $56 \mathrm{~nm}$ for $\mathrm{PMMA}_{72} \mathrm{PS}_{62} \mathrm{SH}$.
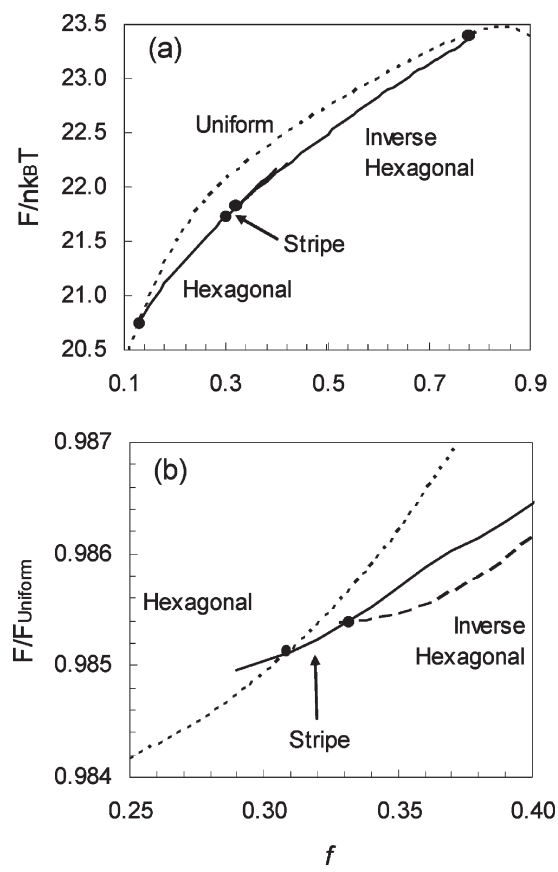

Figure 8. (a) Free energy (F) curves for the four phases in the solventincluded model showing the phase transition points and (b) a magnified region of part a with the free energy curves divided by free energy of the uniform phase.

The Influence of Solvent. In observing the prominent disagreements between the experimental and theoretical results, and the low order of the phase-separated films it became apparent that the initial state of the film may have a significant effect on the structure of the film. In particular, based on the comparison of the $d \mathrm{PS}-\mathrm{PMMA}-\mathrm{OH}$ and PMMA-PS-SH results, the presence of residual solvent in films post washing was hypothesized to have a significant effect. Reports in the literature have noted that both the content of solvent in very thin films is relatively high ${ }^{29}$ and that the solvent can influence phase behavior. ${ }^{30}$

To test this hypothesis, a single SCFT calculation was performed on a tethered diblock copolymer system $\left(L / a N^{1 / 2}=\right.$ $0.45)$ in the presence of solvent. This model used effectively the same method as previously reported, ${ }^{10}$ but differed from the previous model in that two additional interaction parameters (between each of the two polymer blocks and the solvent) replaced the surface tension term $\Lambda N$, i.e. the upper surface is now modeled as a film/solvent rather than a film/ vacuum surface. The parameters chosen for this model were $\chi_{\mathrm{AB}} N=50, \chi_{\mathrm{AS}} N=150, \chi_{\mathrm{BS}} N=100$, where A denotes the upper block, B the lower (grafted) block, and $\mathrm{S}$ the solvent. The latter two were loosely based on the calculated solubility parameters of toluene with $d$ PS and PMMA.

Figure 8 shows the free-energy verses composition plot for this model. Most notably the phase boundaries for this system are significantly shifted relative to those reported in Figure 2, with the phase boundaries being: disordered (uniform)/hexagonal: $f=0.12$; hexagonal/stripe: $f=0.31$; stripe/inverse hexagonal: $f=0.33$; and inverse hexagonal/ uniform: $f=0.79$. These phase boundaries are much more consistent with the results observed for the $d \mathrm{PS}-\mathrm{PMMA}-$ $\mathrm{OH}$ and $\mathrm{PMMA}-\mathrm{PS}-\mathrm{SH}$ films and strongly suggest that this is the cause of the variance observed. The stripe phase also has a relatively small compositional range and its free energy is not significantly different from the two hexagonaltype phases, suggesting that this may be difficult to isolate experimentally. 

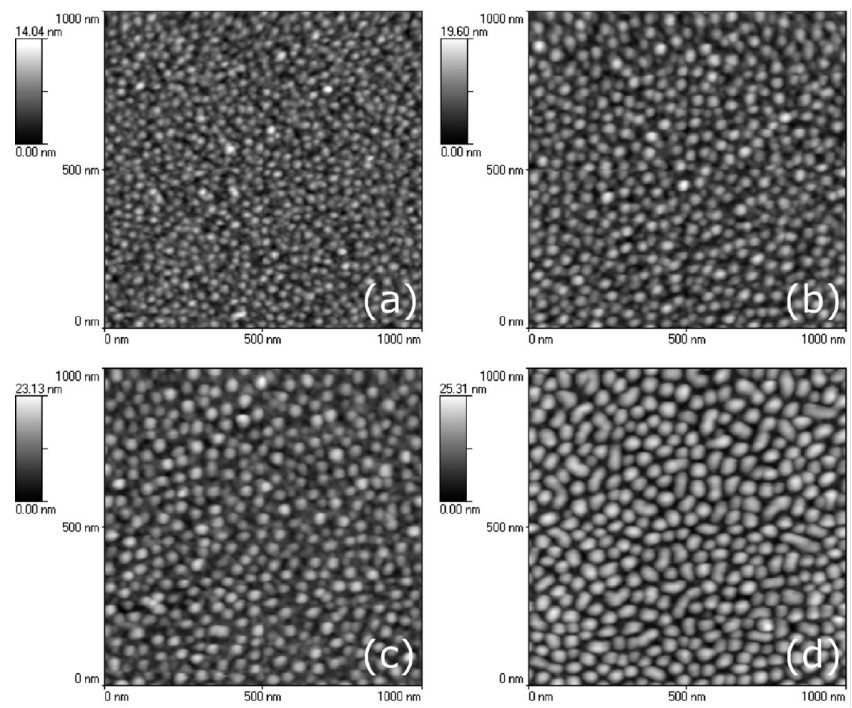

Figure 9. AFM topographic images of (a) $d \mathrm{PS}_{13} \mathrm{PMMA}_{100} \mathrm{OH}$, (b) $d \mathrm{PS}_{33} \mathrm{PMMA}_{122} \mathrm{OH}$, (c) $d \mathrm{PS}_{47} \mathrm{PMMA}_{135} \mathrm{OH}$, and (d) $d \mathrm{PS}_{62} \mathrm{PMMA}_{69^{-}}$ $\mathrm{OH}$ solvent exchanged with acetone.

In the experimental systems, the films do not show significant microphase separation after washing and subsequently further annealing is required. For the PMMA-PS-SH, it is easy to see that the solvent content of the films remain high throughout the annealing process, leading to the observed variation between these films and the previously published "dry" film model. For the $d \mathrm{PS}-\mathrm{PMMA}-\mathrm{OH}$ films the situation is somewhat more complex as it is expected that the solvent will be driven off during the annealing process. Notably though, the presence of residual solvent is expected to both lower the glass transition temperature of each polymer block and enhance chain mobility. ${ }^{29}$

For the $d \mathrm{PS}-\mathrm{PMMA}-\mathrm{OH}$ films, we propose that at the start of the second thermal annealing process, when there is still solvent in the film, the film phase separates in accordance with the solvent-included phase diagram. After a period of time the solvent is removed from the film by the high temperature and low pressure conditions inside the oven. This reduces the mobility of the polymer chains, making reorganization of the polymer slower and effectively leaves the film in a kinetically trapped, nonequilibrium state. The observed phases will therefore be closely related to the solvent-included phases predicted above, though no solvent will be present in the film.

Formation of the "dry"-state phases is conceivably possible, but very long annealing times are likely to be required to achieve this. Alternatively, the use of highly volatile neutral solvents, for instance supercritical $\mathrm{CO}_{2}$, ${ }^{31}$ in the washing process may facilitate the formation of the "dry" phases.

Given the success of these initial solvent-included SCFT calculations, we shall be expanding this work and a more complete model will be published in the future.

Samples Treated by Solvent Exchange. Along with the thermal annealing experiments various $d$ PS- $b$-PMMA $-\mathrm{OH}$ and PMMA- $b$-PS-SH samples were treated by a solvent exchange process using cyclohexane (selective for PS) or acetone (selective for PMMA). ${ }^{32}$ These experiments are similar to those performed by Zhao et al. ${ }^{11,12}$ and Usov et al. ${ }^{32}$ but differ in that these films were prepared using the graftingto rather than grafting-from technique; subsequently, the films reported here are thinner and have lower grafting-densities than those previously reported.

AFM images (Figure 9) of the $d$ PS- $b$-PMMA-OH samples treated with acetone (Supporting Information), showed strong
Table 3. Pre-Treatment Film Thicknesses $(L)$ and Grafting Densities $(\sigma)$ of the $d$ PS- $b$-PMMA-OH Films Treated by Solvent Exchange with Acetone (Subscript A) and Cyclohexane (Subscript C)

\begin{tabular}{lrrrc}
\hline \multicolumn{1}{c}{ polymer } & \multicolumn{1}{c}{$L_{\mathrm{A}}(\mathrm{nm})$} & $\sigma_{\mathrm{A}}\left(\mathrm{nm}^{-2}\right)$ & \multicolumn{1}{c}{$L_{\mathrm{C}}(\mathrm{nm})$} & $\sigma_{\mathrm{C}}\left(\mathrm{nm}^{-2}\right)$ \\
\hline$d \mathrm{PS}_{13} \mathrm{PMMA}_{100} \mathrm{OH}$ & $8.72 \pm 0.03$ & 0.054 & $12.99 \pm 0.04$ & 0.080 \\
$d \mathrm{PS}_{33} \mathrm{PMMA}_{122} \mathrm{OH}$ & $11.42 \pm 0.03$ & 0.051 & $13.04 \pm 0.03$ & 0.058 \\
$d \mathrm{PS}_{47} \mathrm{PMMA}_{135} \mathrm{OH}$ & $11.46 \pm 0.04$ & 0.043 & $13.59 \pm 0.04$ & 0.051 \\
$d \mathrm{PS}_{62} \mathrm{PMMA}_{69} \mathrm{OH}$ & $14.29 \pm 0.04$ & 0.074 & $14.57 \pm 0.03$ & 0.075
\end{tabular}

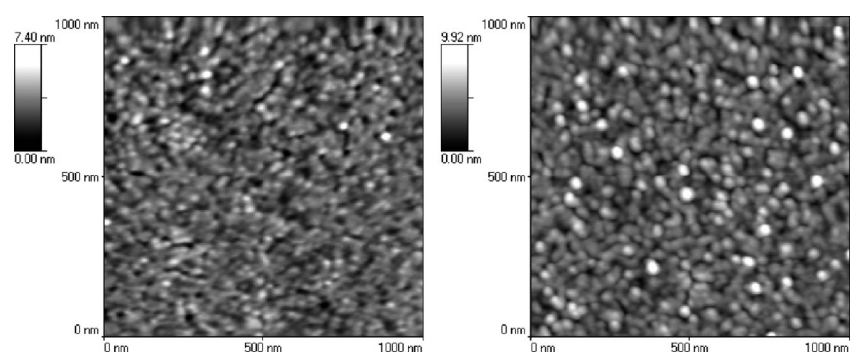

Figure 10. AFM topographic images of $\mathrm{PMMA}_{52} \mathrm{PS}_{108} \mathrm{SH}$ (left) and $\mathrm{PMMA}_{72} \mathrm{PS}_{31} \mathrm{SH}$ (right) solvent exchanged with cyclohexane.

texturing of the polymer film in line with observations by Zhao et al. ${ }^{12}$ This texturing of the surface is attributed to the precipitation of small aggregates of the upper block $(d \mathrm{PS})$, while the lower block (PMMA), remains solubilized. ${ }^{11}$ Upon drying of the films in air, the solvated block will subsequently collapse around the already precipitated upper block, leading to the observed structures.

As with the phase separation behavior examined above the size of these aggregates will be limited by the grafting density of the polymer. Notably, as is shown by the $d \mathrm{PS}_{47} \mathrm{PMMA}_{135} \mathrm{OH}$ and $d \mathrm{PS}_{62} \mathrm{PMMA}_{69} \mathrm{OH}$ samples, where the former has a smaller $d$ PS block but a much larger PMMA block, the size of the upper block has an apparently greater influence on the size of the aggregates. The average radii of the aggregates were calculated by particle analysis to be: $d \mathrm{PS}_{13} \mathrm{PMMA}_{100} \mathrm{OH}=$ $15.0 \mathrm{~nm}, d \mathrm{PS}_{33} \mathrm{PMMA}_{122} \mathrm{OH}=17.8 \mathrm{~nm}, d \mathrm{PS}_{47} \mathrm{PMMA}_{135} \mathrm{OH}=$ $20.0 \mathrm{~nm}$, and $d \mathrm{PS}_{62} \mathrm{PMMA}_{69} \mathrm{OH}=20.9 \mathrm{~nm}$.

Along with this lateral size increase the height of the particles also increases significantly with increasing $f$, with the average aggregate heights being 7.2, 11.1, 12.9, and $15.4 \mathrm{~nm}$, respectively. These are approximately equal to the presolvent exchange height as measured by ellipsometry (Table 3), however as the volume of the film must be conserved this implies that there is a contiguous layer of polymer underlying the particles that is not imaged by AFM. Unfortunately, due to the roughness of the textured films it is not possible to get an accurate estimate of the average film thickness using ellipsometry or other techniques.

In an attempt to verify the structure of the aggregates, phase images of the above samples were collected along with the topographic images (Supporting Information). Unfortunately these phase images show noticeable shadowing effects from the pronounced topographic features and so no definite conclusions could be drawn.

Solvent exchange experiments (using cyclohexane) were also performed with the PMMA- $b$-PS-SH films listed in Table 2 (annealed morphologies shown in Figure 7). Some of these films showed texturing (Figure 10 and Supporting Information), which was similar to the texturing observed with the $d \mathrm{PS}-b$-PMMA-OH films shown in Figure 9. However, as with the solvent annealed films, it appears that the underlying roughness of the gold layer has as impact on the behavior of the films with the aggregates in the PMMA- $b$ PS-SH films displaying much greater variation in size in a 

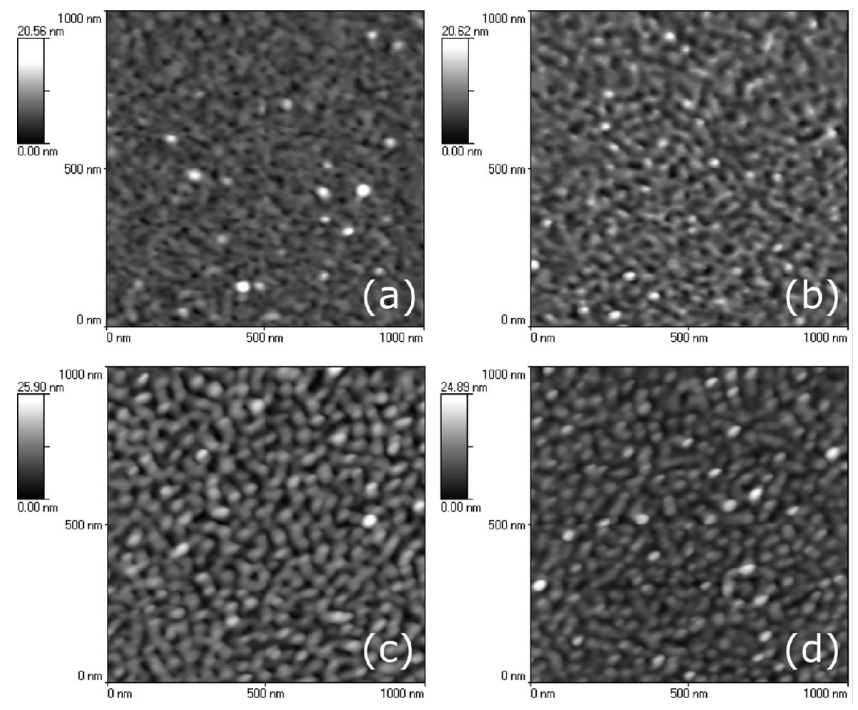

Figure 11. AFM topographic images of (a) $d \mathrm{PS}_{13} \mathrm{PMMA}_{100} \mathrm{OH}$, (b) $d \mathrm{PS}_{33} \mathrm{PMMA}_{122} \mathrm{OH}$, (c) $d \mathrm{PS}_{47} \mathrm{PMMA}_{135} \mathrm{OH}$, and (d) $d \mathrm{PS}_{62} \mathrm{PMMA}_{69^{-}}$ $\mathrm{OH}$ solvent exchanged with cyclohexane.

given image than those observed in the $d \mathrm{PS}-b$-PMMA-OH films.

The complementary experiments to those described above were performed on various $d \mathrm{PS}-b$-PMMA-OH samples using cyclohexane (Figure 11). For these films, as $f$ increases there is a progressive shift from a nearly homogeneous layer to an arrangement of largely hemispherical aggregates, while FFT analysis of the images gave periodicities of $42 \mathrm{~nm}$ for $d \mathrm{PS}_{13} \mathrm{PMMA}_{100} \mathrm{OH}, 46 \mathrm{~nm}$ for $d \mathrm{PS}_{33} \mathrm{PMMA}_{122} \mathrm{OH}$, and $50 \mathrm{~nm}$ for both $d \mathrm{PS}_{47} \mathrm{PMMA}_{135} \mathrm{OH}$ and $d \mathrm{PS}_{62} \mathrm{PMMA}_{69} \mathrm{OH}$.

We believe that this structure variation with $f$ is caused by the increased asymmetry of the system when it has a solvated upper block and an unsolvated lower block. In the bulk state, it is well-known that increasing the asymmetry of the diblock copolymer leads to an increase in the curvature of the interface between the phase-separated structures. ${ }^{33}$ For grafted systems, the picture is somewhat more complex and the tension induced by increasing the asymmetry of the system in favor of the upper block (for instance by swelling with solvent) may be partially relieved through extension of the solvated chain away from the substrate. However, if the asymmetry is sufficiently large, the entropic cost associated with this extension will become large enough that curvature of the interface will also occur.

We propose therefore that the near-homogeneous topography observed in the $d \mathrm{PS}_{13} \mathrm{PMMA}_{100} \mathrm{OH}$ film occurs because the short $d \mathrm{PS}$ block in this polymer can extend relatively easily and therefore significant curvature of the interface is not required. Conversely, for the $d \mathrm{PS}_{62} \mathrm{PMMA}_{69} \mathrm{OH}$ film, chain extension of the large $d$ PS block is entropically less favorable, and subsequently the interface has greater curvature leading to the observed hemispherical aggregates.

Following drying of the film, the upper solvated layer would collapse largely evenly onto the already precipitated lower block, thereby preserving the underlying structure through the whole film. Again the phase images (Supporting Information) of these samples give no strong indication of the location of the different polymer species in the film and so this hypothesis cannot be confirmed.

For both the cyclohexane and acetone exchanged $d \mathrm{PS}-b$ PMMA-OH films the structures formed were not thermally stable and subsequently were destroyed by a short period of annealing at $180^{\circ} \mathrm{C}$ (Supporting Information).

\section{Conclusions}

In this study, the microphase separation behavior of tethered diblock copolymer films was examined and the results compared with recent theoretical calculations in an attempt to validate them. In qualitative agreement with the theory, it was observed that the phase present in the films changed from disordered/ uniform, to hexagonal, to inverse hexagonal and finally to uniform again as the fraction of the upper block increased. The fourth predicted phase, the stripe phase, was also observed in small areas in some of the samples.

Quantitatively however the results were markedly different from those produced in the published phase diagram for dry brushes and it was hypothesized that the observed phase behavior was influenced by residual solvent in the films prior to and during the second annealing process. This was supported by new, preliminary calculations on the tethered block copolymer system with included solvent, which generated phase boundaries much more consistent with the experimental results. For the films treated with a thermal annealing process it was proposed that the films initially phase-separate into to their solvent-included phases and that, upon evaporation of the solvent, the films become kinetically trapped, thereby preserving the symmetry and domain size of the solvent-included phase structures. A more detailed study of the solvent-included phase diagrams will be the subject of future work.

Under solvent exchange conditions the $d$ PS- $b$-PMMA-OH films showed strong aggregation consistent with previous work. Using PMMA-selective acetone, hemispherical structures were observed (with the size again being dependent on the size of the upper block), while with $d$ PS-selective cyclohexane the shape of the aggregates was dependent on the polymer composition.

Acknowledgment. This work was supported by EPSRC grants EP/G026203/1 and EP/F029616/1 to I.W.H. and M.W. M., and EP/E015220/1 to support V.L. The authors thank Prof. Joe Keddie (University of Surrey) for the kind use of his ellipsometer and Dr. James Nobbs (University of Leeds) for his support of V.L.

Supporting Information Available: Figures showing AFM line scans of the PMMA- $b$-PS-SH samples, and AFM images of the annealed but unwashed $d \mathrm{PS}-b$-PMMA-OH samples, solvent exchanged $d$ PS- $b$-PMMA-OH samples (before and after heating), and solvent exchanged PMMA- $b$-PS-SH samples and text giving the calculation of "typical" $\Delta \gamma$ values. This material is available free of charge via the Internet at http://pubs.acs.org.

\section{References and Notes}

(1) Hamley, I. W., The Physics of Block Copolymers; Oxford University Press: Oxford, U.K., 1998.

(2) Matsen, M. W.; Bates, F. S. Macromolecules 1996, 29 (4), 1091-1098.

(3) Segalman, R. A. Mater. Sci. Eng. R 2005, 48 (6), 191-226.

(4) Hamley, I. W. Prog. Polym. Sci. 2009, 34 (11), 1161-1210.

(5) Cohen Stuart, M. A.; Huck, W. T. S.; Genzer, J.; Muller, M.; Ober, C.; Stamm, M.; Sukhorukov, G. B.; Szleifer, I.; Tsukruk, V. V.; Urban, M.; Winnik, F.; Zauscher, S.; Luzinov, I.; Minko, S. Nat. Mater. 2010, 9 (2), 101-113.

(6) Liu, D.; Wang, T.; Keddie, J. L. Langmuir 2009, 25 (8), 4526-4534.

(7) Fasolka, M. J.; Mayes, A. M. Annu. Rev. Mater. Res. 2001, 31 (1), $323-355$.

(8) Akgun, B.; Ugur, G.; Brittain, W. J.; Majkrzak, C. F.; Li, X.; Wang, J.; Li, H.; Wu, D. T.; Wang, Q.; Foster, M. D. Macromolecules 2009, 42 (21), 8411-8422.

(9) Wang, J.; Müller, M. Macromolecules 2009, 42 (6), 2251-2264

(10) Matsen, M. W.; Griffiths, G. H. Eur. Phys. J. E 2009, 29 (2), 219-227.

(11) Zhao, B.; Brittain, W. J.; Zhou, W.; Cheng, S. Z. D. Macromolecules 2000, 33 (23), 8821-8827.

(12) Zhao, B.; Brittain, W. J.; Zhou, W.; Cheng, S. Z. D. J. Am. Chem. Soc. 2000, 122 (10), 2407-2408.

(13) Kakwere, H.; Perrier, S. b. J. Am. Chem. Soc. 2009, 131 (5), 1889-1895. 
(14) Whittaker, M. R.; Goh, Y.-K.; Gemici, H.; Legge, T. M.; Perrier, S.; Monteiro, M. J. Macromolecules 2006, 39 (26), 9028-9034.

(15) Gemici, H.; Legge, T. M.; Whittaker, M.; Monteiro, M. J.; Perrier, S. J. Polym. Sci. A: Polym. Chem. 2007, 45 (11), 2334-2340.

(16) Konkolewicz, D.; Gray-Weale, A.; Perrier, S. b. J. Am. Chem. Soc. 2009, 131 (50), 18075-18077.

(17) Tanaka, K.; Takahara, A.; Kajiyama, T. Macromolecules 1996, 29 (9), 3232-3239.

(18) Russell, T. P. Macromolecules 1993, 26 (21), 5819.

(19) Zhao, Y.; Sivaniah, E.; Hashimoto, T. Macromolecules 2008, 41 (24), 9948-9951.

(20) Fetters, L. J.; Lohse, D. J.; Richter, D.; Witten, T. A.; Zirkel, A. Macromolecules 1994, 27 (17), 4639-4647.

(21) Mark, J. E., Ed. Physical Properties of Polymers Handbook, 2nd ed.; Springer: New York, 2007.

(22) Jeong, U.; Kim, H.-C.; Rodriguez, R. L.; Tsai, I. Y.; Stafford, C. M.; Kim, J. K.; Hawker, C. J.; Russell, T. P. Adv. Mater. 2002, 14 (4), 274-276.

(23) O’Driscoll, B. M. D.; Oren, R.; Hamley, I. W. Polym. Adv. Techol. 2010, in press.
(24) Chen, Y.; Huang, H.; Hu, Z.; He, T. Langmuir 2004, 20 (9), 38053808 .

(25) Wang, D.; Fujinami, S.; Nakajima, K.; Nishi, T. Macromolecules 2010, 43 (7), 3169-3172.

(26) Krausch, G.; Hipp, M.; Boeltau, M.; Marti, O.; Mlynek, J. Macromolecules 1995, 28 (1), 260-263.

(27) Sivaniah, E.; Hayashi, Y.; Matsubara, S.; Kiyono, S.; Hashimoto, T.; Kukunaga, K.; Kramer, E. J.; Mates, T. Macromolecules 2005, 38 (5), 1837-1849.

(28) Turner, M. S.; Joanny, J. F. Macromolecules 1992, 25 (24), 6681-6689.

(29) Garcia-Turiel, J.; Jerome, B. Colloid Polym. Sci. 2007, 285 (14), $1617-1623$.

(30) Zhang, X. H.; Berry, B. C.; Yager, K. G.; Kim, S.; Jones, R. L.; Satija, S.; Pickel, D. L.; Douglas, J. F.; Karim, A. ACS Nano 2008, 2 (11), 2331-2341.

(31) Russell, T. P. Personal communication, 2010

(32) Usov, D.; Gruzdev, V.; Nitschke, M.; Stamm, M.; Hoy, O.; Luzinov, I.; Tokarev, I.; Minko, S. Macromolecules 2007, 40 (24), 8774-8783.

(33) Matsen, M. W.; Bates, F. S. Macromolecules 1996, 29 (23), 7641-7644. 